\title{
Neoadjuvant therapy of BRCA1-driven ovarian cancer by combination of cisplatin, mitomycin $\mathrm{C}$ and doxorubicin
}

\author{
Tatiana V. Gorodnova ${ }^{1}$, Anna P. Sokolenko ${ }^{1,2}$, Khristina B. Kotiv ${ }^{1}$, Tatiana N. Sokolova', Alexandr O. Ivantsov ${ }^{1,2}$, \\ Konstantin D. Guseynov ${ }^{1}$, Ekaterina A. Nekrasova', Olga A. Smirnova ${ }^{1}$, Igor V. Berlev ${ }^{1,3}$ and Evgeny N. Imyanitov ${ }^{1,2,3^{*}}$
}

\begin{abstract}
Background: Cisplatin, mitomycin C and anthracyclines demonstrate high activity in BRCA1-deficient tumors. This study aimed to evaluate the efficacy of the triplet combination of these drugs in BRCA1-driven high-grade serous ovarian carcinomas (HGSOCs).

Methods: Ten HGSOC patients with germ-line BRCA1 mutation received neoadjuvant chemotherapy (NACT) consisting of mitomycin C $10 \mathrm{mg} / \mathrm{m}^{2}$ (day 1), doxorubicin $30 \mathrm{mg} / \mathrm{m}^{2}$ (days 1 and 8) and cisplatin $80 \mathrm{mg} / \mathrm{m}^{2}$ (day 1), given every 4 weeks (MAP regimen). The comparator group included 16 women, who received standard NACT combination of paclitaxel $175 \mathrm{mg} / \mathrm{m}^{2}$ and carboplatin (6 AUC), given every 3 weeks (TCbP scheme).

Results: None of the patients treated by the MAP scheme demonstrated complete pathologic response in ovaries, while 4 women showed absence of tumor cells in surgically excised omental specimens. When chemotherapy response scores (CRS) were considered, poor responsiveness (CRS 1) was not observed in the MAP group, but was common for the TCbP regimen (6/16 (38\%) for ovaries and 5/16 (31\%) for omentum; $p=0.05$ and 0.12, respectively). Median treatment-free interval (TFI) was not reached in women treated by the MAP, but was 9.5 months for the TCbP scheme $(p=0.1)$. The rate of the recurrence within 1 year after the completion of the treatment was 4/10 (40\%) for the MAP and 10/13 (77\%) for the TCbP $(p=0.1)$.

Conclusions: The attempt to intensify NACT by administering combination of 3 drugs did not result in high rate of complete pathologic responses. However, there was a trend towards higher efficacy of the MAP regimen versus conventional TCbP scheme with regard to CRS and clinical outcomes.
\end{abstract}

Keywords: Ovarian cancer, BRCA1, Neoadjuvant chemotherapy, Cisplatin, Mitomycin C, Doxorubicin

\section{Background}

Ovarian cancer $(\mathrm{OC})$ is a common malignancy, which holds the leading position in the mortality caused by gynecological tumors [1]. The worldwide incidence of OC approaches approximately three hundred thousand new cases per year, with almost two-thirds of affected

\footnotetext{
* Correspondence: evgeny@imyanitov.spb.ru

${ }^{1}$ N.N. Petrov Institute of Oncology, 197758 Saint-Petersburg, Russia

${ }^{2}$ St.-Petersburg Pediatric Medical University, 194100 Saint-Petersburg, Russia

Full list of author information is available at the end of the article
}

patients dying from this disease [2]. High-grade serous ovarian cancer (HGSOC) is the most frequent OC histological type. A significant portion of HGSOCs is attributed to germ-line mutations in BRCA1 or BRCA2 genes. BRCA1/2-driven ovarian tumors usually develop via inactivation of the remaining allele of the involved gene. Consequently, these cancers demonstrate a tumorselective deficiency in DNA repair by homologous recombination and pronounced sensitivity to platinum compounds, PARP inhibitors and mitomycin C $[3,4]$. 
Ovarian tumors often do not cause symptoms at early stages; therefore, most HGSOC patients are diagnosed with already inoperable disease. These women are often subjected to neoadjuvant chemotherapy (NACT), which is aimed to reduce tumor burden and allow surgical intervention [5]. BRCA1-associated ovarian malignancies demonstrate significantly better responses to the NACT as compared to sporadic neoplasms [6]. Although these patients usually undergo complete cytoreductive surgery followed by adjuvant therapy, most BRCA1-driven HGSOCs eventually relapse [7]. These relapses are attributed to the acquisition of the resistance of tumor clones to systemic therapy. The most known mechanism of acquired platinum resistance is the emergence of mutations, which restore the open reading frame in the $B R C A 1$ gene [4]. This route is mainly applicable to heavily pretreated patients but appears to be less characteristic for the initial phases of OC therapy [8]. On the other hand, NACT often results in the selection of BRCA1proficient cells, which exist in small amounts in chemonaive tumors and repopulate tumor mass during platinum exposure [9].

Intensification of the therapy is a common approach aimed to prevent the emergence of resistant clones. We have previously reported promising results of applying cisplatin plus mitomycin $\mathrm{C}$ combination for the NACT of BRCA1-driven carcinomas. This therapy resulted in a significant reduction of the tumor burden in all analyzed patients and in complete pathologic responses observed in $2 / 12(17 \%)$ treated women [10]. We reasoned that combining this regimen with an additional drug may further improve the outcomes of NACT. Previous studies suggested that BRCA1-driven tumors are particularly sensitive to anthracyclines, while their responsiveness to taxanes is under the question $[10,11]$. Consequently, we decided to add doxorubicin to cisplatin plus mitomycin $\mathrm{C}$ as a third drug. Here we present the results of the trial involving this 3 -drug combination.

\section{Methods}

The design of the study was discussed on the council involving medical oncologists, cancer gynecologists and hereditary cancer specialists. It was decided that the pilot trial would include 10 patients with initially inoperable BRCA1-driven HGSOC, and the main end-point will be the rate of pathologic complete responses. While all patients received the same neoadjuvant regimen (MAP: mitomycin C $10 \mathrm{mg} / \mathrm{m}^{2}$ (day 1), doxorubicin $30 \mathrm{mg} / \mathrm{m}^{2}$ (days 1 and 8), cisplatin $80 \mathrm{mg} / \mathrm{m}^{2}$ (day 1 ), given every 4 weeks), the physicians were permitted to administer the therapy of their choice after the surgery. This approach provided some flexibility given that the combination of carboplatin and paclitaxel (TCbP) is a standard option for the treatment of ovarian cancer [1] and that some
post-NACT tumor samples have restored BRCA1 function and therefore may not be potentially responsive to platinum drugs $[8,9]$. The recruitment of patients was performed from August 2017 to December 2018 based on the results of the PCR-based test for Slavic recurrent germ-line mutations [12, 13]. According to the study protocol, all tumor samples were subjected to the lossof-heterozygosity (LOH) analysis before the NACT and after the surgery. LOH test was performed as described in [9]. All tumors were also analyzed for the TP53 somatic mutations, given that TP53 inactivation is a ubiquitous feature of BRCA1-driven carcinomas [14]. The study was approved by the local Ethics Committee. All patients included in the study provided informed consent.

Although this study was not randomized, we considered for the comparison of treatment outcomes 16 consecutive patients with germ-line BRCA1 mutations, who were referred to the N.N. Petrov Institute of Oncology (St.-Petersburg, Russia) between February 2017 and December 2019 and were subjected to a standard NACT combination of paclitaxel $175 \mathrm{mg} / \mathrm{m}^{2}$ plus carboplatin (6 AUC), given every 3 weeks. Most of these patients were negative for PCR-detectable recurrent BRCA1 mutations; however, they were found to carry a germ-line pathogenic allele upon the analysis of the entire BRCA1 and BRCA2 coding sequence, i.e., after the start of NACT [13].

All women receiving MAP or TCbP were managed by the same surgical team. Tumor responses were evaluated according to RECIST criteria using computed tomography and magnetic resonance imaging. None of the patients treated by MAP or TCbP received bevacizumab. Three patients in the MAP arm but none in the TCbP group were subjected to the hyperthermic intraperitoneal chemotherapy (HIPEC) during surgery. None of the included patients described in this report received maintenance by PARP inhibitors after completion of the firstline therapy, as this indication was not approved in Russia at the time of the study.

The statistical analysis was performed using SPSS 13.0 software package. Age distribution and the duration of the follow-up were compared by the Mann-Whitney Utest. Median treatment-free interval (TFI) was evaluated using Kaplan-Meyer curves. Other comparisons were performed with the Fisher's exact test.

\section{Results}

Five patients included in the study of the neoadjuvant combination of mitomycin $\mathrm{C}$, cisplatin and doxorubicin had stage IIIC HGSOC and another 5 women were diagnosed with stage IV disease (Table 1). Partial response to this therapy was observed in all 10 cases considered. Seven women had toxicities of grades 1 or 2; 2 patients had 


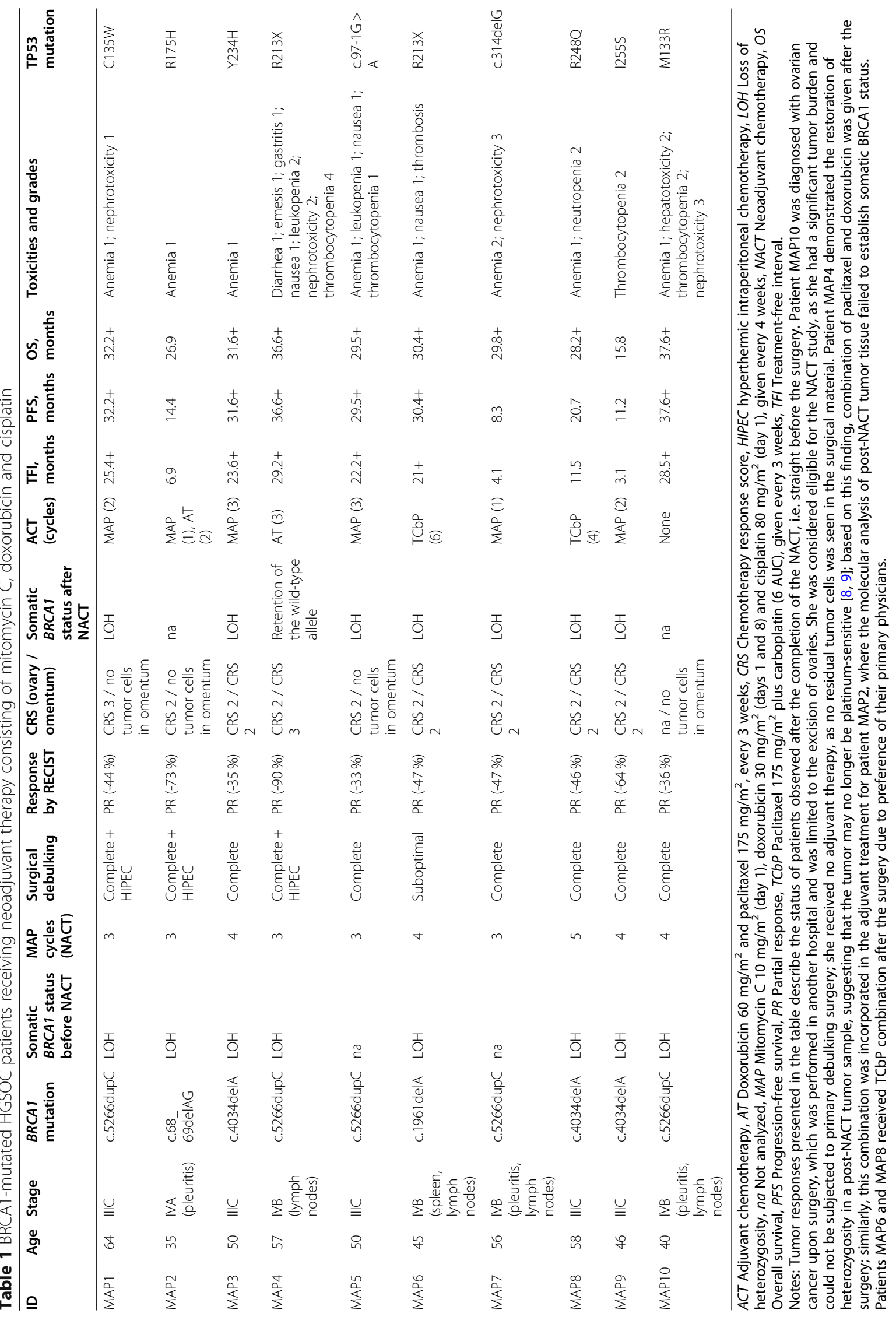


Table 2 Comparative characteristics of patients with BRCA1-mutated HGSOC receiving NACT combination of mitomycin C, doxorubicin and cisplatin versus women treated by paclitaxel plus carboplatin

\begin{tabular}{|c|c|c|c|}
\hline Clinical characteristics & MAP group $(N=10)$ & TCbP group $(N=16)$ & Statistical comparison \\
\hline $\begin{array}{l}\text { Median age of onset } \\
\text { (range) }\end{array}$ & $50(35-64)$ & $49(37-72)$ & Not significant \\
\hline $\begin{array}{l}\text { Pattern of } B R C A 1 \\
\text { mutations }\end{array}$ & $\begin{array}{l}\text { c.5266dupC }(n=5), \text { c.4034delA } \\
(n=3), \text { c.68_69delAG }(n=1), \\
\text { c.1961delA }(n=1)\end{array}$ & 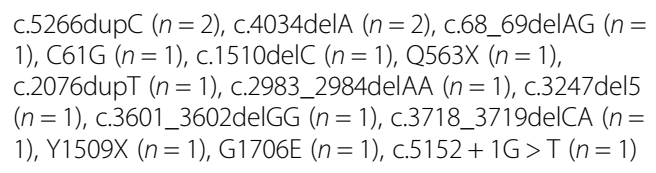 & $\begin{array}{l}p=0.005 \text { (founder vs. non- } \\
\text { founder mutations; Fisher's } \\
\text { exact test) }\end{array}$ \\
\hline \multicolumn{4}{|l|}{ FIGO stage } \\
\hline 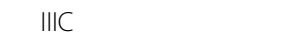 & $5(50 \%)$ & $12(75 \%)$ & \multirow{4}{*}{$\begin{array}{l}p=0.23 \text { (stage III vs. IV; } \\
\text { Fisher's exact test) }\end{array}$} \\
\hline IVA & $1(10 \%)$ & $2(13 \%)$ & \\
\hline IVB & $4(40 \%)$ & $2(13 \%)$ & \\
\hline NACT cycles (range) & $3-5$ & $3-8$ & \\
\hline \multicolumn{4}{|l|}{ Cytoreduction } \\
\hline Optimal & $9(90 \%)$ & $14(88 \%)$ & \multirow[t]{3}{*}{$p=1.0$ (Fisher's exact test) } \\
\hline Suboptimal & $1(10 \%)$ & $1(6 \%)$ & \\
\hline None & 0 & $1(6 \%)$ & \\
\hline \multicolumn{4}{|l|}{ Response by RECIST } \\
\hline$C R$ & 0 & 0 & \multirow{4}{*}{$\begin{array}{l}p=0.12 \text { (objective response } \\
\text { vs. lack of objective response } \\
\text { Fisher's exact test) }\end{array}$} \\
\hline PR & 10 (100\%) & $11(69 \%)$ & \\
\hline SD & 0 & $4(25 \%)$ & \\
\hline PD & 0 & $1(6 \%)$ & \\
\hline \multicolumn{4}{|c|}{ Chemotherapy response score (CRS) in the ovaries } \\
\hline CRS 1 & 0 & $6(38 \%)$ & \multirow{4}{*}{$\begin{array}{l}p=0.05 \text { (CRS } 1 \text { vs. other; } \\
\text { Fisher's exact test) }\end{array}$} \\
\hline CRS 2 & $8(80 \%)$ & $9(56 \%)$ & \\
\hline CRS 3 & $1(10 \%)$ & 0 & \\
\hline $\begin{array}{l}\text { Tissue not available } \\
\text { for evaluation }\end{array}$ & $1(10 \%)$ & $1(10 \%)$ & \\
\hline \multicolumn{4}{|c|}{ Chemotherapy response score (CRS) in the omentum } \\
\hline CRS 1 & 0 & $5(31 \%)$ & \multirow{6}{*}{$\begin{array}{l}p=0.12 \text { (CRS } 1 \text { vs. other; } \\
\text { Fisher's exact test) }\end{array}$} \\
\hline CRS 2 & $5(40 \%)$ & $8(50 \%)$ & \\
\hline CRS 3 & $1(10 \%)$ & 0 & \\
\hline No tumor cells & $4(40 \%)$ & $2(13 \%)$ & \\
\hline $\begin{array}{l}\text { Tissue not available } \\
\text { for evaluation }\end{array}$ & 0 & $1(6 \%)$ & \\
\hline ACT cycles (range) & $1-6$ & $1-6$ & \\
\hline $\begin{array}{l}\text { Median follow-up, } \\
\text { months (range) }\end{array}$ & $30.1(15.8-36.6)$ & $23.4(10.7-45.2)$ & $p=0.28$ (Mann-Whitney Test) \\
\hline $\begin{array}{l}\text { Median treatment- } \\
\text { free interval }(95 \% \mathrm{Cl})\end{array}$ & Not reached & $9.5(7.8-11.2)$ & $\begin{array}{l}p=0.109 \text { (Log Rank [Mantel- } \\
\text { Cox]) }\end{array}$ \\
\hline $\begin{array}{l}\text { Recurrence within } \\
\text { one year after } \\
\text { completion of } \\
\text { treatment }\end{array}$ & $4(40 \%)$ & $10 / 13^{\mathrm{a}}(77 \%)$ & $p=0.1$ (Fisher's exact test) \\
\hline
\end{tabular}

ACT adjuvant chemotherapy, CRS chemotherapy response score, MAP mitomycin C $10 \mathrm{mg} / \mathrm{m}^{2}$ (day 1), doxorubicin $30 \mathrm{mg} / \mathrm{m}^{2}$ (days 1 and 8 ), cisplatin $80 \mathrm{mg} / \mathrm{m}^{2}$ (day 1), given every 4 weeks, NACT neoadjuvant chemotherapy, TCbP paclitaxel $175 \mathrm{mg} / \mathrm{m}^{2}$ plus carboplatin (6 AUC), given every 3 weeks

${ }^{\mathrm{a}} 13$ out of 16 patients had sufficient follow-up for the estimation of 1-year recurrence rate 
toxicity grade 3 and 1 subject experienced grade IV thrombocytopenia. None of the HGSOCs showed a complete pathological response in the ovaries, and only one woman demonstrated chemotherapy response score (CRS) 3, according to Böhm et al. [15]. Omental tumor response, which is more predictive for the disease outcome than adnexal CRS [15], showed considerably better values: 4 women had no residual tumor cells in the omentum, 1 patient had CRS 3 and 5 cases demonstrated CRS 2. There were no instances of poor responsiveness to the therapy categorized as CRS 1 . The median TFI and progressionfree survival (PFS) were not reached.

Sixteen patients receiving paclitaxel plus carboplatin had slightly more favorable stage distribution, as 12 subjects had HGSOC of stage IIIC and 4 patients demonstrated stage IV disease (Table 2). While all patients treated by MAP showed partial response, 4/16 (25\%) women subjected to the TCbP combination produced only the disease stabilization and there was one HGSOC with the progression on this therapy. There was a remarkable difference from MAP regimen with regard to pathological responses, as minimal response score was observed in 6/16 (38\%) cases for ovarian tumor masses and 5/16 (31\%) HGSOCs for omental metastases $(p=0.05$ and 0.12 , respectively). While the median follow-up for the TCbP group was shorter than for MAP patients, median TFI was already achieved and reached 9.5 months (Fig. 1). Thirteen patients had sufficient follow-up to evaluate 1-year outcomes; the recurrence rate at 1 year after the completion of the treatment was 10/13 (77\%) for the TCbP, while the same value was $4 / 10$ $(40 \%)$ for the MAP regimen $(p=0.1)$.

\section{Discussion}

Our previous study involving 12 BRCA1-mutated HGSOCs treated with the combination of cisplatin and mitomycin $\mathrm{C}$ revealed complete pathologic responses in 2 out of 12 patients [10]. We anticipated that the addition of doxorubicin to this combination may increase the rate of elimination of all tumor cells detectable in surgically excised tissues. The obtained data are sufficient to state that the applied triplet does not significantly increase the rate of complete pathologic responses as compared to the previously applied combination of two drugs.

At the same time, short-term results of MAP therapy look encouraging. In addition to a reasonably good rate of objective responses, half of the included cases demonstrated complete or nearly-complete absence of tumor cells in the omentum. Omental response score is the main predictor of the long-term outcomes of NACT, so it is a valuable marker allowing robust evaluation of various chemotherapy regimens [15].

Previous studies suggested that the TCbP regimen may be less efficient in BRCA1-mutated HGSOCs as compared to other NACT schemes [10]. These data sets compared prospective and retrospective patients treated by different surgeons. The quality of surgical debulking is critical for the outcome of HGSOC treatment, therefore these comparisons are prone to biases. Within the present study, we analyzed groups of patients who were managed at the same time interval by the same group of surgeons. However, the MAP and TCbP groups of patients were not balanced with regard to the pattern of mutations. The selection of patients for the MAP therapy involved rapid PCR tests for recurrent Slavic mutations [13]. The TCbP

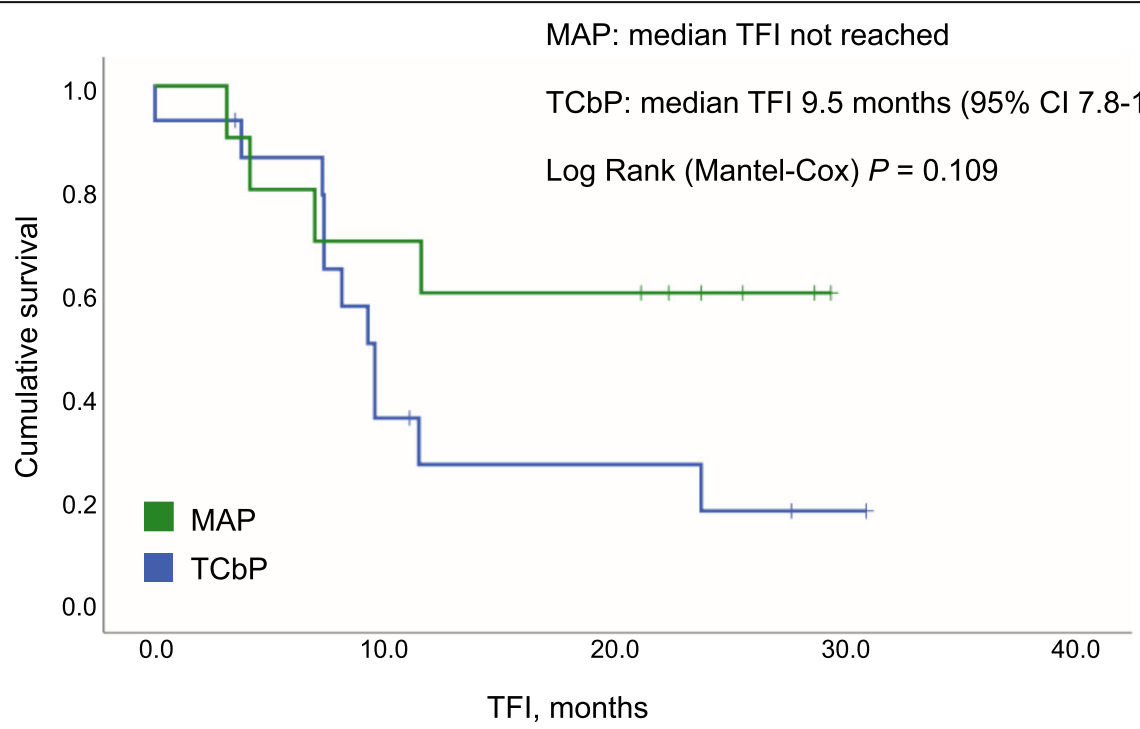

Fig. 1 Treatment-free interval for patients treated with the combination of mitomycin C, cisplatin and doxorubicin and for women treated by the paclitaxel plus carboplatin doublet 
HGSOC group is significantly enriched by subjects with "rare" BRCA1 pathogenic alleles, which were detected by the next-generation sequencing analysis after the start of NACT. There are some data suggesting that distinct $B R C A 1$ and BRCA2 mutations may exert distinct sensitivity to platinum compounds and PARP inhibitors [4]. Although we acknowledge differences in the pattern of BRCA1 mutations as a limitation of the study, it should be noted that all published trials on PARP inhibitors did not consider the type of mutation as a confounding factor $[4,16]$.

The landscape of the treatment BRCA1/2-mutated HGSOC is rapidly evolving. In particular, PARP inhibitors have been recently included in the standards for the first-line maintenance therapy, as they significantly delay the time to tumor recurrence [16]. None of the patients considered in this report received PARP inhibitors because they were not locally approved for early lines of HGSOC treatment at the time of the study. Consequently, it is unclear whether the differences observed between distinct NACT regimens will be maintained upon the incorporation of PARP-targeted drugs.

\section{Conclusions}

In summary, this study suggests that BRCA1-associated HGSOCs may require distinct therapeutic NACT regimens as compared to conventional TCbP doublet. If this is the case, the fast turn-around time for $B R C A 1 / 2$ testing could become a critical factor for appropriate treatment decisions. Recent data indicate that BRCA1/2associated HGSOCs do not show inferior outcomes when treated by NACT before the surgery, while primary surgical intervention is clearly the best approach in sporadic ovarian tumors $[7,17,18]$. These findings are likely to increase the acceptance of NACT for BRCA1/2 germline mutation carriers and, therefore, stimulate large neoadjuvant clinical trials for this category of HGSOC patients.

\section{Abbreviations}

CRS: Chemotherapy response score; HGSOC: High-grade serous ovarian cancer; LOH: Loss of heterozygosity; MAP: Mitomycin C, anthracycline, platinum; NACT: Neoadjuvant chemotherapy; OC: Ovarian cancer; PFS: Progression-free survival; TCbP: Taxanes and carboplatin; TFI: Treatmentfree interval
}

\section{Authors' contributions}

TG, KK, IB, KG, EN and OS managed study patients and analyzed clinical data; TS performed the molecular analysis; Al carried out morphological analysis; AS integrated and analyzed the obtained data and contributed to the manuscript preparation; El designed the study and wrote the first draft of the manuscript. All authors have read and approved the final version of the manuscript.

\section{Funding}

This work has been supported by the Ministry of Science and Higher Education of the Russian Federation (grant № 075-15-2020-789).

\section{Availability of data and materials}

The datasets used and/or analyzed during the current study are available from the corresponding author on reasonable request.

Ethics approval and consent to participate

The study was performed in full accordance with ethics guidelines. A written informed consent from study participants was obtained.

\section{Consent for publication}

All living patients provided the consent for publication.

\section{Competing interests}

The authors declare that they have no competing interests.

\section{Author details}

${ }^{1}$ N.N. Petrov Institute of Oncology, 197758 Saint-Petersburg, Russia.

${ }^{2}$ St.-Petersburg Pediatric Medical University, 194100 Saint-Petersburg, Russia.

${ }^{3}$ I.I. Mechnikov North-Western Medical University, 195067 St.-Petersburg,

Russia.

Received: 15 January 2021 Accepted: 27 January 2021

Published online: 03 February 2021

\section{References}

1. Lheureux S, Gourley C, Vergote I, Oza AM. Epithelial ovarian cancer. Lancet. 2019:393:1240-53.

2. Bray F, Ferlay J, Soerjomataram I, Siegel RL, Torre LA, Jemal A. Global cancer statistics 2018: GLOBOCAN estimates of incidence and mortality worldwide for 36 cancers in 185 countries. CA Cancer J Clin. 2018;68:394-424.

3. Iyevleva AG, Imyanitov EN. Cytotoxic and targeted therapy for hereditary cancers. Hered Cancer Clin Pract. 2016;14:17.

4. Le Page C, Amuzu S, Rahimi K, Gotlieb W, Ragoussis J, Tonin PN. Lessons learned from understanding chemotherapy resistance in epithelial tuboovarian carcinoma from BRCA1and BRCA2mutation carriers. Semin Cancer Biol. 2020;19:S1044-579X(20)30177-2.

5. Leary A, Cowan R, Chi D, Kehoe S, Nankivell M. Primary surgery or neoadjuvant chemotherapy in advanced ovarian cancer: The debate continues. Am Soc Clin Oncol Educ Book. 2016;35:153-62.

6. Gorodnova TV, Sokolenko AP, Ivantsov AO, lyevleva AG, Suspitsin EN, Aleksakhina SN, et al. High response rates to neoadjuvant platinum-based therapy in ovarian cancer patients carrying germ-line BRCA mutation. Cancer Lett. 2015;369:363-7.

7. Gorodnova T, Sokolenko A, Ni V, Ivantsov A, Kotiv K, Petrik S, et al. BRCA1associated and sporadic ovarian carcinomas: outcomes of primary cytoreductive surgery or neoadjuvant chemotherapy. Int J Gynecol Cancer. 2019:29:779-86.

8. Sokolenko AP, Bizin IV, Preobrazhenskaya EV, Gorodnova TV, Ivantsov AO, lyevleva AG, et al. Molecular profiles of BRCA1-associated ovarian cancer treated by platinum-based therapy: Analysis of primary, residual and relapsed tumors. Int J Cancer. 2020;146:1879-88.

9. Sokolenko AP, Savonevich EL, Ivantsov AO, Raskin GA, Kuligina ES, Gorodnova TV, et al. Rapid selection of BRCA1-proficient tumor cells during neoadjuvant therapy for ovarian cancer in BRCA1 mutation carriers. Cancer Lett. 2017;397:127-32.

10. Gorodnova TV, Kotiv KB, Ivantsov AO, Mikheyeva ON, Mikhailiuk Gl, Lisyanskaya AS, et al. Efficacy of neoadjuvant therapy with cisplatin plus mitomycin C in BRCA1-mutated ovarian cancer. Int J Gynecol Cancer. 2018; 28:1498-506.

11. Byrski T, Gronwald J, Huzarski T, Grzybowska E, Budryk M, Stawicka M, et al. Pathologic complete response rates in young women with BRCA1positive breast cancers after neoadjuvant chemotherapy. J Clin Oncol. 2010;28:375-9.

12. Cybulski C, Kluźniak W, Huzarski T, Wokołorczyk D, Kashyap A, Rusak B, et al. The spectrum of mutations predisposing to familial breast cancer in Poland. Int J Cancer. 2019;145:3311-20.

13. Sokolenko AP, Sokolova TN, Ni VI, Preobrazhenskaya EV, lyevleva AG, Aleksakhina SN, et al. Frequency and spectrum of founder and non-founder BRCA1 and BRCA2 mutations in a large series of Russian breast cancer and ovarian cancer patients. Breast Cancer Res Treat. 2020;184:229-35. 
14. Holstege $H$, Joosse SA, van Oostrom CT, Nederlof PM, de Vries A, Jonkers J. High incidence of protein-truncating TP53 mutations in BRCA1-related breast cancer. Cancer Res. 2009;69:3625-33.

15. Böhm S, Faruqi A, Said I, Lockley M, Brockbank E, Jeyarajah A, et al.

Chemotherapy response score: Development and validation of a system to quantify histopathologic response to neoadjuvant chemotherapy in tuboovarian high-grade serous carcinoma. J Clin Oncol. 2015;33:2457-63.

16. Madariaga A, Lheureux S, Oza AM. Tailoring ovarian cancer treatment: Implications of BRCA1/2 mutations. Cancers (Basel). 2019;11:416.

17. Petrillo M, Marchetti C, De Leo R, Musella A, Capoluongo E, Paris I, et al. BRCA mutational status, initial disease presentation, and clinical outcome in high-grade serous advanced ovarian cancer: a multicenter study. Am J Obstet Gynecol. 2017;217:334.e1-e9.

18. Ponzone R. BRCA1/2 status and chemotherapy response score to tailor ovarian cancer surgery. Crit Rev Oncol Hematol. 2020;157:103128.

\section{Publisher's Note}

Springer Nature remains neutral with regard to jurisdictional claims in published maps and institutional affiliations.

Ready to submit your research? Choose BMC and benefit from:

- fast, convenient online submission

- thorough peer review by experienced researchers in your field

- rapid publication on acceptance

- support for research data, including large and complex data types

- gold Open Access which fosters wider collaboration and increased citations

- maximum visibility for your research: over $100 \mathrm{M}$ website views per year

At $\mathrm{BMC}$, research is always in progress.

Learn more biomedcentral.com/submissions 